\title{
UNIVERSIDAD PARA MAYORES: REINVENTARSE E INNOVAR EN TIEMPOS PANDÉMICOS
}

\author{
Senior University: Reinvention and Innovation in a Pandemic Context
}

Recibido: 17 de junio de 2021

Aceptado: 22 de octubre de 2021

María Isabel Torres Cazorla

Profesora Titular de Derecho Internacional

Público y Relaciones Internacionales

mtorres@uma.es

Universidad de Málaga

\section{RESUMEN}

La presente contribución aborda la experiencia práctica obtenida en tiempos de pandemia, respecto a la continuidad de una actividad relacionada con el ejercicio y disfrute del Derecho a la Educación a lo largo de toda la vida, en lo que se conoce como el Aula de Mayores de la Universidad. Recibe diversas denominaciones en las distintas instituciones universitarias de nuestro entorno, estando enmarcada en la labor social que la Universidad ha de cumplir; esta actividad tiene como objetivo primordial que personas de una determinada edad (normalmente de los 50-55 años en adelante) puedan recibir conocimientos sobre materias de su interés mediante cursos formativos, cuya duración es también variable. El aspecto fundamental de esta actividad radica en ofrecer a este colectivo una oportunidad para seguir aprendiendo (en ocasiones, acercarse a las aulas universitarias por vez primera) sobre temas diversos de su elección. La posibilidad de socializar es otro factor clave de la Universidad para Mayores. La pandemia de la COVID-19 ha ocasionado que estos cursos, su modelo, su forma de impartición e inclusive sus participantes (tanto el profesorado como los propios alumnos) hayan debido adaptarse a esta nueva realidad. Este trabajo analiza la experiencia vivida por la autora en el Aula de Mayores+55 de la Universidad de Málaga durante el curso académico 2020/21, así como ofrece algunos datos de otros entornos universitarios de nuestro país, y de las alternativas que se han planteado para no interrumpir esta actividad. De ahí el título de esta contribución, que alude a la necesidad de renovarse e innovar en tiempos pandémicos.

\section{PALABRAS CLAVE}

Aula de Mayores, COVID-19, derecho a la educación, educación a lo largo de toda la vida, ODS 4. 


\section{ABSTRACT}

This article explains the practical experience of her author as a consequence of the pandemic at the socalled Senior University (Aula de Mayores +55 ) of the University of Málaga. Lifelong learning opportunities for all, together with the right to education in a pandemic context is a key point. The Senior University is an instrument for the social projection of the Universities and also has different names (depending of the University). This is an opportunity provided to people aged 50-55 years and over, to expand their knowledge about different subjects (Sanitary questions, Social and Legal Sciences, Literature, Arts, Economics, Foreign Languages, digital skills, among others). This is an opportunity, coming for the first time to the University (or coming back) as a lifelong socialization and learning experience. The Senior University has had to be adapted to the pandemic context (a new experience for students and teachers, developed in most cases via on-line tools). The personal experience of the author at the University of Málaga (Aula de Mayores+55) is the primary aspect to be considered, including an analysis of the practice followed by Spanish Universities. The personal motto is reinvention and innovation in a pandemic context.

\section{KEYWORDS}

COVID-19, lifelong learning, right to education, SDG 4, Senior University. 
Sumario: 1. Introducción: visión global, en tiempos pre-pandémicos. 2. Y llegó la pandemia, el estado de alarma, el confinamiento...y todo saltó por los aires. 3. ¿Quo Vadis, Aula de Mayores+55, durante el curso 2020/21? 4. El Aula de Mayores en la Universidad española en tiempos pandémicos: análisis de la práctica. 5. El Aula de Mayores a la luz del ODS 4: ajustes y desajustes en tiempos de COVID-19. 5.1. El ODS 4 confrontado con el aprendizaje durante toda la vida. 5.2. Los efectos colaterales de la pandemia para el colectivo de mayores y su formación. 6. A modo de colofón, ante una realidad en transición. 7. Referencias bibliográficas y otros documentos de consulta.

\section{Introducción: visión global, en tiempos pre-pandémicos.}

Este trabajo constituye una reflexión que tiene como fundamento el relato de una experiencia, tras participar en el Aula de Mayores de la Universidad de Málaga, en estos tiempos pandémicos que la COVID-19 ha traído consigo. La ineludible adaptación de esta docencia, teniendo como telón de fondo el ODS 4 y el aprendizaje durante toda la vida, son aspectos esenciales que se abordarán en este estudio. En la Universidad de Málaga (UMA, en adelante), el Aula de Mayores se instauró hace varias décadas, y está dirigida a mayores de 55 años que quieren ampliar conocimientos en muy diversos ámbitos. Este Aula de Mayores (o senior, dirigida a un colectivo que oscila entre los 4055 años en adelante, en las diferentes Universidades), tiene denominaciones diversas. La más común a lo largo de nuestra geografía nacional suele ser Aula para Mayores (a la que se añadió hace unos años en la UMA la referencia a +55 para hacer partícipes en esta actividad a ese colectivo a partir de esa edad) ${ }^{1}$. También se la conoce como Aula Permanente de Formación Abierta, Aula de la Experiencia, Programa Universitario para Mayores, Universidad Senior, Programa UNED Senior, etc., dependiendo de la universidad concreta a la que estemos haciendo referencia, como se verá más adelante ${ }^{2}$.

En suma, cabe destacar que la puesta en práctica de un Programa Universitario para Mayores en numerosas universidades españolas es un fenómeno que encuentra su punto de partida en la segunda mitad de los años noventa del pasado siglo (Gairín, Rodríguez Gómez, Armengol y del Arco, 2013, 64), y que hoy constituye una actividad plenamente consolidada.

La experiencia del Aula de Mayores de la UMA permite volver la vista atrás, a los cursos académicos 2000/01, 2001/02 y 2002/03. La participación de los profesores implicados en esta labor por aquel entonces consistía en la impartición de una conferencia de una hora y media de duración, en cada uno de los cursos académicos en que se participaba. Esta actividad se llevaba a cabo ante un auditorio amplio, publicándose después en un volumen conjunto un resumen de dichas conferencias (Torres Cazorla 2001, 181-188; 2002, 293-299 y 2003, 403-408), que versaban sobre diversos ámbitos del conocimiento. El objetivo del Aula de Mayores era -y continúa siendo así- formar a este colectivo de personas adultas en diversos temas de su interés (que podían abarcar cuestiones de índole general o específica sobre Historia, Geografía, Arte, Literatura, Filosofía, Música, Ciencias, Biología, Psicología, Periodismo, Astronomía, Medicina, Economía, Derecho, Salud, Nutrición, Idiomas, Informática, u otros temas ofertados por el profesorado que deseaba participar en esta experiencia). Si bien el conjunto de cursos ofertados dedicados al Derecho es reducido, los alumnos están muy interesados en las

\footnotetext{
${ }^{1}$ Véase toda la información en https://www.uma.es/aula-de-mayores/. Este vínculo web, y todos los que se mencionan en el presente trabajo han sido consultados por última vez el día 30 de septiembre de 2021.

${ }^{2}$ Una denominación que se diferencia un tanto de las demás en nuestro entorno es la que se ha dado a esta experiencia en la Universidad de Valencia: la Nau Gran. Como puede verse en https://www.uv.es/naugran/es/nau-gran.html.
} 
cuestiones de actualidad, dado que suelen estar muy bien informados, y los temas jurídicos que les afectan directamente o inciden en nuestro país y entorno cercanos, les resultan apasionantes.

Años más tarde, el Aula de Mayores de la UMA cambió su pauta de funcionamiento, introduciéndose un nuevo formato de cursos monográficos, que se desarrollan durante un trimestre. Estos cursos siguen igualmente una pauta de formación en múltiples ámbitos, decantándose por una oferta muy variada de cursos que el alumno elige, siendo la mayoría de ellos de carácter trimestral y algunos otros anuales (por ejemplo, los talleres de idiomas e informática). En esa modalidad, desde el año 2014, la autora de estas líneas participa como docente en un curso de esta índole en la sede de Málaga, así como impartiendo conferencias en el segundo semestre en distintos pueblos de la provincia, actividad esta última que conforma el segundo eje de actuación de la Universidad en distintas poblaciones. El curso trimestral guarda relación con el Derecho Internacional Público, y consiste en una sesión semanal durante el primer trimestre, de hora y media de duración. Cabe destacar que el curso se imparte durante dos días a la semana, desde octubre hasta diciembre, y el segundo día semanal es impartido por un docente de la Facultad de Económicas (el profesor Francisco Calderón). Dicho curso se titula "Claves para entender el mundo actual: una aproximación a través del Derecho Internacional y la Economía". Ello explica esa división en dos sesiones semanales, cada una de las cuales se dedica a uno de estos aspectos: Derecho Internacional, por un lado, y Economía, como segundo eje de análisis.

Las sesiones tienen una hora y media de duración, y están dedicadas cada una de ellas a explicar un tema, en numerosas ocasiones relacionado directamente con la realidad internacional del momento. Son sesiones muy participativas, que ofrecen a los alumnos la posibilidad de preguntar y cuestionar lo que se les explica, incluso de proponer temas concretos de su interés para sesiones posteriores. Todo ello se ha llevado a cabo utilizando diversas herramientas que permiten ofrecer elementos docentes atractivos (Presentaciones PowerPoint, documentales, páginas web, documentos adicionales para profundizar, artículos de prensa, etc.). Una de las grandezas del Aula de Mayores es su carácter de enseñanza no reglada ni sometida a exámenes por parte del alumnado, lo que permite adaptarnos al interés del propio alumnado, sin perjuicio del previo diseño de un plan docente de contenidos mínimos, diseñado y presentado a los futuros estudiantes en el momento de matricularse.

Cabe destacar lo valioso que resulta participar como docente en el Aula de Mayores, una de las mejores experiencias que puede tener un profesor universitario, sin lugar a dudas. Es lugar común para quienes han tenido ocasión de impartir docencia en este entorno, llegar a una serie de conclusiones que son enormemente positivas: los alumnos están muy motivados, tienen un gran interés en aprender, son enormemente participativos y suelen ser un grupo numeroso, pero sin que ello sea una barrera que impida o entorpezca su participación. Sin duda, esta experiencia formativa constituye un elemento más que aporta calidad de vida (Moreno Crespo y Cruz Díaz 2012, 1530) a las personas que se involucran en ese lifelong learning. Destaca ese proceso de retroalimentación, como experiencia docente, que sirve además como incentivo que permite aprender y socializar a un colectivo que se caracteriza por su experiencia vital y sus deseos de formarse en ámbitos muy distintos.

Esta introducción trata de contextualizar la experiencia que relataremos, comenzando por explicar lo acontecido el curso académico 2019/2020, antes de que se desatase la pandemia de la COVID-19, que cambió el mundo para todos, y también lo hizo para los participantes en el Aula de Mayores+55 (docentes y discentes). Dicho curso académico 
(en la asignatura del primer trimestre), eran 46 los estudiantes matriculados en la misma. Además, en ese mismo curso 2019/2020, en diciembre, se realizó con dichos alumnos una actividad que la propia UMA promovió, en el contexto de la asignatura: una visita a la oficina de CIFAL Málaga ${ }^{3}$, centro de UNITAR ${ }^{4}$, de Naciones Unidas en la ciudad de Málaga. Los alumnos del Aula de Mayores+55 tuvieron la oportunidad de escuchar una conferencia y asistir a un taller en el centro CIFAL, relacionado con los Objetivos de Desarrollo Sostenible (ODS) y la Agenda 2030. Fue una sesión formativa, en la que pudieron conocer de primera mano la existencia de este centro, desconocido para muchos, así como trabar contacto con la realidad de los ODS. Todo ello tuvo lugar en diciembre de 2019, un par de meses antes de que la pandemia de coronavirus con sus letales consecuencias, se extendiese por todo el planeta. Esta era nuestra realidad entonces, donde el Aula de Mayores se definía como una fórmula de aprendizaje integral y socialización para este colectivo (Valle Aparicio, 2014, 135).

El punto de partida previo a la COVID-19 se caracterizaba por unas clases presenciales, participativas, donde el aprendizaje durante toda la vida en las materias elegidas y la socialización, eran elementos esenciales. Todo ello cambiaría radicalmente a consecuencia de la pandemia y las restricciones que llevó aparejadas.

\section{Y llegó la pandemia, el estado de alarma, el confinamiento...y todo saltó por} los aires.

La expansión mundial del virus nos ha dado un baño de realidad, enormemente duro, a todos los habitantes del planeta. Y el Aula de Mayores no podía permanecer ajena a esta situación. Todas las actividades previstas a partir de mediados de marzo de $2020^{5}$ en el Aula de Mayores (tanto en la sede de Málaga como en la provincia ${ }^{6}$ ), tuvieron que suspenderse, dado que las mismas eran de carácter presencial. En un contexto en que todos estábamos confinados era imposible llevar a cabo dicha actividad, o al menos realizar una adaptación de esa presencialidad tradicional a otros escenarios, de una manera inmediata. Ello provocó la suspensión inicial y cancelación posterior de las actividades durante ese segundo y tercer trimestre del año 2020. Esta situación ha continuado durante el curso 2020/2021, al menos en este formato de conferencias en

\footnotetext{
${ }^{3}$ Dichas siglas corresponden a Centro Internacional de Formación de Autoridades y Líderes (CIFAL), y concretamente la Oficina de Málaga, creada en 2018, suministra información en https://www.cifalmalaga.org/web/sobre-cifal-malaga/.

${ }^{4}$ Dichas siglas corresponden al Instituto de Naciones Unidas para la Formación Profesional e Investigación (UNITAR), véase https://unitar.org/.

${ }^{5}$ El Real Decreto 463/2020, de 14 de marzo, por el que se declara el estado de alarma para la gestión de la situación de crisis sanitaria ocasionada por la COVID-19 fue la norma que fundamentó la adopción de estas medidas iniciales, algunas de cuyas disposiciones han sido declaradas inconstitucionales por sentencia del Tribunal Constitucional 148/2021, de 14 de julio (véase BOE núm. 67, de 14 de marzo de 2020, y núm. 182, de 31 de julio de 2021, publicando este último la sentencia mencionada). Esta fue solamente una de las múltiples normas que se adoptaron en diversos ámbitos para tratar de frenar la pandemia, pudiendo consultarse para ello en el $B O E$ la sección dedicada a las normas que guardan relación con este tema, y a las que remitimos: https://www.boe.es/biblioteca_juridica/index.php?tipo=C.

${ }^{6}$ En años anteriores, la autora impartió conferencias de esta índole, en el contexto del Aula de Mayores, en localidades como Vélez-Málaga, Fuengirola, Marbella o Nerja. Esta actividad consistía en impartir 4 conferencias a lo largo de dos semanas, en colaboración con la profesora Elena del Mar García Rico, profesora de Derecho Internacional Público de la Universidad de Málaga, distribuyéndonos las sesiones, atendiendo a la agenda de cada una de nosotras y a los temas, que solían ser "La sociedad internacional en el siglo XXI"; "Naciones Unidas: retos y oportunidades", "España en la Unión Europea: un largo noviazgo", y "Los derechos de los mayores: una asignatura pendiente".
} 
diferentes sedes provinciales, si bien manteniéndose cierta actividad (virtual) en la sede de Málaga, como se explicará a continuación.

Lamentablemente, esto se ha extendido a las actividades del colectivo de personas de edad, no sólo en lo que respecta al Aula de Mayores en la provincia de Málaga, sino también a numerosos programas dirigidos a ellos que se realizaban por parte de los ayuntamientos, las asociaciones vecinales y otros colectivos. Al ser este grupo poblacional uno de los más afectados inicialmente por la letalidad de la pandemia, la consecuencia lógica ha sido cancelar todo aquello que pudiese provocar una concentración de personas y un eventual contagio masivo. Si esto ha ocurrido de manera generalizada en la población, su reflejo ha sido aún más acusado respecto a las actividades de este colectivo, por razones a las que no podemos permanecer ajenos.

De hecho, una de las actividades esenciales del Aula de Mayores+55 es la socialización entre las personas que conforman este colectivo, de forma general y a través de una Asociación (AMADUMA, Asociación de Mayores de la UMA), donde realizaban viajes y actividades de diversa índole (talleres de arte, teatro, literatura y otros). Por supuesto, la pandemia ha dado al traste con todo ello, al menos de manera transitoria. Dicha asociación tiene su web ${ }^{7}$, desde la que se informa a los miembros de diferentes actividades; recupero una frase de una de sus entradas, animando a todos los socios "a seguir, con resiliencia, y llevando al ánimo de todos el sentirse protagonistas (con la pandemia) de una especie de situación de ciencia ficción, tras la cual se podrán retomar los viajes, las comidas, y las actividades sociales que se realizaban". Ante todo, resiliencia ${ }^{8}$, palabra que sin duda describe como ninguna otra la posición manifestada por buena parte de la población mundial con el objetivo de hacer frente a esta grave situación.

\section{3. ¿Quo vadis, Aula de Mayores+55 durante el curso 2020/21?}

Y llegó la desescalada, y la necesidad de programar el nuevo curso académico que se avecinaba (2020/21), también en el Aula de Mayores+55. A la vista de la incertidumbre reinante, los responsables de esta actividad trataron de prever lo mejor y lo peor frente al nuevo curso. Así, en junio, se invitó a los profesores implicados en esta experiencia el curso anterior a volver a participar en el Aula, y unas semanas más tarde, se preguntó una fórmula previsora- si estarían dispuestos a impartir dicha docencia y, si la misma no podía ser presencial porque las circunstancias sanitarias lo impidiesen, podrían hacerlo en modo on-line.

Seguramente, un número elevado de docentes -entre las que me incluyo- si bien manifestamos nuestro compromiso con el Aula de Mayores, aunque la misma tuviese que desarrollarse en modo on-line, no alentábamos muchas esperanzas acerca de que esa fórmula prosperase. La singularidad de esta docencia y el público al que se dirige la misma hacían surgir dudas razonables acerca de una adaptación posible al modelo online. Si por algo se caracterizan las clases del Aula de Mayores es precisamente por la interacción, el debate entre los alumnos, la participación, en una palabra. Y todo ello resulta -al menos en apariencia- un tanto contrapuesto a las reuniones telemáticas. Además, la necesidad de contar con la tecnología necesaria para poder participar en las

\footnotetext{
${ }^{7}$ Dicha web es http://elblogdeamaduma.blogspot.com/.

${ }^{8}$ Nuestro diccionario de la Real Academia Española define este término como la "capacidad de adaptación de un ser vivo frente a un agente perturbador o un estado o situación adversos" y, en una segunda acepción que también puede ser aplicable a nuestra realidad, como la "capacidad de un material, mecanismo o sistema para recuperar su estado inicial cuando ha cesado la perturbación a la que había estado sometido". Véase https://dle.rae.es/resiliencia.
} 
clases on-line, suponía un segundo inconveniente, al menos a priori. En suma, la docencia on-line exigía (por parte de todos) que nos reinventásemos.

A finales de septiembre de 2020, se nos informó que el primer trimestre del curso sería forzosamente on-line, y que se impartirían los cursos que hubiesen recibido un número mínimo de matriculados (en este caso, 10 personas). Comparando esta cifra con la que solían tener dichos cursos en modo presencial como era habitual, el descenso de participantes era notable. De hecho, 23 cursos (de los 55 que se ofertaban este curso académico durante el primer trimestre) tuvieron que ser cancelados. El curso que imparte quien escribe estas líneas ha contado en esta edición on-line con 17 alumnos matriculados, número elevado, si tenemos en cuenta la singularidad del Aula de Mayores+55, donde esta situación de la virtualidad resultaba novedosa. Además, es sorprendente el hecho de que el primer trimestre salieron adelante 32 cursos; sin duda, ello ha supuesto un esfuerzo económico, organizativo y un reto sin precedentes para alumnos y docentes. Por supuesto, el número de alumnos matriculados de manera general en el Aula de Mayores +55 ha descendido mucho, casi a la mitad, pues no hay que olvidar que uno de los aspectos importantes de esta experiencia ha sido siempre la posibilidad de socializar e intercambiar puntos de vista, que en un escenario virtual es mucho más complicado. Y a ello se suma la brecha tecnológica, que no se debe menospreciar, máxime en un colectivo de edad avanzada, donde el acceso a la tecnología no siempre es fácil.

Ha de destacarse el carácter positivo de esta experiencia, al observar que el alumnado mayor de 55 años se ha sumado a esta iniciativa de la virtualidad, haciendo, de la necesidad virtud, y adoptando -y adaptándose- a las clases on-line, al igual que lo hemos hecho los docentes. En el caso de la asignatura que imparte quien escribe estas líneas, la primera sesión de la misma tuvo lugar el pasado 19 de octubre de 2020, participando en ella 13 alumnos, y solamente una persona manifestó haber tenido problemas para acceder a Google Meet, la aplicación elegida finalmente para impartir las clases. Desde la organización de la UMA se dejó plena libertad a cada docente para utilizar la herramienta que considerase más adecuada para las clases on-line. Google Meet parecía ser -a nuestro juicio, después de utilizar al menos seis herramientas diferentes en diversos contextos docentes desde que se desató la pandemia ${ }^{9}$ - la que ofrecía una posibilidad de conectarse más sencilla, tratando así de evitar problemas mayores y con la intención de facilitar al máximo posible la continuidad de esta actividad, en este contexto complejo. La simplificación ha sido el leif motiv que nos ha guiado, al tratarse de una experiencia novedosa en este marco del Aula de Mayores+55.

El conjunto de las sesiones discurrió fluidamente, contando con la participación activa del alumnado, aspecto satisfactorio dada la incertidumbre reinante. Ésta ha sido la tónica general del trimestre, con lo cual la experiencia ha sido altamente positiva. Los alumnos han hecho uso también del correo electrónico, planteando propuestas temáticas, recibiendo los materiales complementarios utilizados en clase (vínculos web,

\footnotetext{
${ }^{9}$ La proliferación de diversidad de herramientas de video-conferencia como consecuencia de la situación provocada por la pandemia ha hecho que, de no utilizar ninguna o simplemente usar alguna de modo limitado, tanto docentes como discentes hayamos pasado a realizar un uso enormemente amplio de las mismas. Aplicaciones como Skype, Zoom o Hangouts (esta última pasó a denominarse Google Meet), se complementan con Microsoft Teams - con muchos más recursos pero bastante más compleja en cuanto a su utilización, especialmente para alguien no versado en estas lides-, o el denominado en la UMA Seminario Virtual B y C (este último es el que se viene utilizando en muchas Universidades, denominado Blackboard Collaborate, que usualmente permite el acceso desde el campus virtual o como invitado), o Cisco Webex, entre otras. La opción por Google Meet se debió exclusivamente al hecho de que esta herramienta era, al menos en apariencia y ab initio, la más sencilla para llevar a cabo nuestra docencia y para que los alumnos se conectasen sin muchos problemas.
} 
presentaciones de PowerPoint, textos de lectura, entre otros). Su implicación ha sido muy alta, formulando preguntas relacionadas con la realidad internacional actual, temas que les llamaban la atención, dudas diversas, y propuestas temáticas concretas sobre cuestiones de interés. Todo ello, en el contexto virtual en que se desarrollaba la asignatura, con sesiones síncronas de video-conferencia utilizando la herramienta mencionada.

En la tercera sesión ocurrió un incidente de problemas de conexión de alguno de los participantes, que escribió posteriormente un correo electrónico solicitando material para poder conocer el contenido básico de la clase del día en que falló su conexión a Internet. También ha habido alguna anécdota curiosa (que permite verificar la diferencia con lo que ocurre con los alumnos de las enseñanzas regladas de Grado y Postgrado): mientras los alumnos del Aula de Mayores suelen (un 95\% al menos) encender su cámara y micrófono (lo cual da la sensación de que "hay vida al otro lado"), esto es mucho menos frecuente en los alumnos jóvenes. En ese sentido, en el Aula de Mayores+55 un día empezó a sonar música de una de las asistentes, que tenía abierto el micrófono y la radio puesta, siendo esta una anécdota curiosa de las clases síncronas (en directo). En el caso de los alumnos de enseñanzas regladas, mucho menos proclives a encender la cámara, es el profesor quien debe incentivar -que no obligar- a que al menos quienes intervienen preguntando (de viva voz, no solamente en el chat), enciendan la cámara, aunque sea un momento. Es fundamental, al menos que algunos de los intervinientes lo hagan, para dar la sensación de que no se le está hablando a la pantalla, sin más. En este sentido, se observa una gran diferencia entre el alumnado senior, que no tiene problema alguno en mostrar su imagen e intervenir, frente al alumnado más joven, que se muestra más reticente a ello.

El feedback ofrecido por los participantes en el Aula de Mayores+55 acerca de este sistema on-line muestra una aceptación positiva respecto a esta modalidad alternativa de enseñanza, al menos en momentos complicados donde, de no haber sido así, se hubiesen suspendido todas las actividades del Aula de Mayores en la UMA, como sucedió en la segunda mitad del curso 2019/2020. Dos de los participantes manifestaron que estaban familiarizados con estas tecnologías (ya lo estaban, al tener hijos y familiares en el extranjero y realizar video-conferencias asiduamente); algunos otros señalaron que vivían en otros municipios de la provincia y que si el sistema de enseñanza no hubiese sido online, no se hubiesen matriculado. Estos aspectos hacen ver que la enseñanza virtual también tiene su público, constituyendo una oportunidad para continuar con esta actividad, en tiempos críticos.

El Aula de Mayores+55 y las actividades futuras han evolucionado al ritmo de la pandemia. En el segundo y tercer trimestre de este curso 2020/21 se han cancelado 65 cursos, puesto que la docencia ha seguido siendo on-line y ha sido necesario establecer ese número mínimo de diez participantes para que el curso pudiese impartirse de manera efectiva. En sentido contrario, se han llevado a cabo durante este período (en ese formato on-line) 24 cursos, lo que indica que esa tendencia de que el alumnado siga participando en el Aula de Mayores, aunque sea de forma no presencial, parece consolidarse como tendencia. 


\section{El Aula de Mayores en la Universidad española en tiempos pandémicos: análisis de la práctica}

En las líneas anteriores se han dibujado los cambios y la evolución necesaria que el Aula de Mayores +55 ha experimentado a consecuencia de la pandemia, relatando una experiencia docente desarrollada durante el curso 2020/21. Al hilo de la misma, realizaremos a continuación una comparativa en la que ofreceremos una panorámica de estas experiencias en las Universidades españolas, partiendo de la información básica que suministra la página web de la Asociación Estatal de Programas Universitarios para Mayores (AEPUM) ${ }^{10}$, en la que están inscritas las Universidades socias españolas (tanto públicas como privadas).

Basándonos en dicha información desglosaremos en distintos cuadros explicativos los datos más relevantes que esta iniciativa ha experimentado durante el curso académico 2020/21. Dicha clasificación de las Universidades obedece a la fórmula elegida por cada una de ellas para desarrollar la actividad del Aula de Mayores durante el curso académico que acabamos de dejar atrás (virtual, presencial, fórmula mixta, aplazamiento/cancelación del Aula de Mayores u otras fórmulas imaginativas para continuar con la actividad).

Cabe destacar que la información que se detalla a continuación se ha obtenido investigando en cada uno de los programas del Aula de Mayores de las Universidades españolas socias, elaborando las tablas explicativas que aparecen a continuación con el objetivo de ilustrar de manera más gráfica los aspectos abordados (solución pandémica en cada una de las Universidades socias, nombre y web del programa, así como edad de sus destinatarios). No siempre ha sido fácil obtener la información (especialmente la relativa a las soluciones adoptadas durante el curso 2020/21 a causa de la evolución de la situación sanitaria, así como la edad mínima para participar en estos cursos). En el caso de cada Universidad se ha creado un hipervínculo para que el lector pueda acceder al programa de la institución de que se trate, permitiendo así la posibilidad de indagar en el mismo y ver sus aspectos fundamentales. Todo ello, partiendo de la base de que todas las Universidades, pese a utilizar fórmulas diversas, han tenido en cuenta la evolución de la situación sanitaria, con carácter prioritario.

a) Universidades que se han decantado por la fórmula virtual (síncrona) de impartición de la docencia en el Aula de Mayores (22 Universidades de un total de 49 entidades socias de AEPUM):

\begin{tabular}{|l|c|c|}
\hline $\begin{array}{c}\text { Nombre } \\
\text { Universidad }\end{array}$ & Nombre del Programa & $\begin{array}{c}\text { Edad mínima } \\
\text { matriculados }\end{array}$ \\
\hline \begin{tabular}{l|c|} 
Almería \\
Autónoma de \\
Barcelona
\end{tabular} & $\underline{\text { La Universitat a l'Abast - La Universidad Al }}$ & 55 años \\
\hline $\begin{array}{l}\text { Autónoma } \\
\text { Madrid }\end{array}$ & $\underline{\text { Universidad de Mayores }}$ & 50 años \\
\hline Burgos & $\underline{\text { Título Propio Programa Universidad para los }}$ & 55 años \\
\hline
\end{tabular}

\footnotetext{
${ }^{10}$ Véase Inicio - AEPUM | Asociación Estatal de Programas Universitarios para Mayores. En dicho enlace se puede acceder a dicha información acerca de la propia Asociación, así como al conjunto de Universidades socias de la misma, donde se encuentra también la UMA, concretamente en el vínculo https://www.aepum.es/universidades-socias/.
} 


\begin{tabular}{|c|c|c|}
\hline $\begin{array}{l}\text { Carlos III } \\
\text { Madrid }\end{array}$ & $\underline{\text { UC3M Senior }}$ & 55 años \\
\hline $\begin{array}{l}\text { Castilla La } \\
\text { Mancha }\end{array}$ & $\frac{\text { Programa Universitario José Saramago "50 }}{\text { plus" }}$ & 50 años \\
\hline $\begin{array}{l}\text { Complutense } \\
\text { de Madrid }\end{array}$ & Universidad para los Mayores & 55 años \\
\hline Córdoba & Centro Intergeneracional Francisco Santisteban & 50 años \\
\hline Extremadura & $\frac{\text { Programa Universitario de Mayores de }}{\text { Extremadura }}$ & Personas adultas \\
\hline Islas Baleares & Universitat Oberta per a Majors & $\begin{array}{c}\text { Mayores de } 50 \text { años o } \\
\text { prejubilados con } \\
\text { estudios primarios }\end{array}$ \\
\hline Málaga & $\underline{\text { Aula de Mayores }+55}$ & 55 años \\
\hline $\begin{array}{l}\text { Miguel } \\
\text { Hernández de } \\
\text { Elche }\end{array}$ & $\underline{\text { Aulas Universitarias de la Experiencia }}$ & 55 años \\
\hline Murcia & Aula Senior & 50 años \\
\hline Oviedo & $\frac{\text { Programa Universitario para Mayores de la }}{\text { Universidad de Oviedo }}$ & 50 años \\
\hline $\begin{array}{l}\text { Pablo Olavide } \\
\text { de Sevilla }\end{array}$ & Aula Abierta de Mayores & 50 años \\
\hline $\begin{array}{l}\text { Politécnica de } \\
\text { Cartagena }\end{array}$ & $\underline{\text { Universidad de Mayores }}$ & 50 años \\
\hline $\begin{array}{l}\text { Politécnica de } \\
\text { València }\end{array}$ & $\underline{\text { Universidad Sénior }}$ & 55 años \\
\hline $\begin{array}{l}\text { Pontificia de } \\
\text { Comillas }\end{array}$ & Universidad de Mayores & 50 años \\
\hline Ramon Llull & Programa Universitari per a Gent Gran & Mayores \\
\hline Sevilla & Aula de la Experiencia & 50 años \\
\hline UNED Senior & $\underline{\text { UNED SENIOR }}$ & 55 años \\
\hline $\begin{array}{l}\text { Universitas } \\
\text { Seniorivus } \\
\text { CEU }\end{array}$ & Programa completo de Humanidades & 40 años \\
\hline
\end{tabular}

Tabla núm. 1 (Elaboración propia)

Esta es la posibilidad por la que se han decantado un número mayor de Universidades, atendiendo a la experiencia obtenida en el curso 2019/2020, cuando la enseñanza reglada (Grados y Postgrados) hubo de pasar forzosamente a la virtualidad. Es la fórmula utilizada por la Universidad Autónoma y la Complutense de Madrid, la Universidad de Sevilla y Córdoba, la UNED, entre otras, así como Universidades de tamaño medio como la de Málaga, y otras de ciudades más pequeñas como la de Elche.

b) Universidades que han continuado con la actividad presencial en el Aula de Mayores durante el curso 2020/21 (un total de 10 Universidades): 


\begin{tabular}{|c|c|c|}
\hline $\begin{array}{c}\text { Nombre } \\
\text { Universidad }\end{array}$ & Nombre del Programa & $\begin{array}{l}\text { Edad mínima } \\
\text { matriculados }\end{array}$ \\
\hline Cádiz & Aula Universitaria de Mayores & 55 años \\
\hline Cantabria & Programa Sénior & 50 años \\
\hline Deusto & $\frac{\text { DeustoBide - Escuela de Ciudadanía de la }}{\text { Universidad de Deusto }}$ & Cualquier edad \\
\hline Girona & $\frac{\text { Aules d'Extensió Universitària per a la }}{\text { Gent Gran }}$ & 50 años \\
\hline Granada & $\underline{\text { Aula Permanente de Formación Abierta }}$ & 50 años \\
\hline Jaén & Programa Universitario de Mayores & 55 años \\
\hline La Laguna & $\frac{\text { Estudios Universitarios para Personas }}{\text { Adultas y Mayores }}$ & 45 años \\
\hline $\begin{array}{l}\text { Las Palmas de Gran } \\
\text { Canaria }\end{array}$ & Peritia et Doctrina & 55 años \\
\hline Santiago & IV Ciclo & 50 años \\
\hline Vigo & $\underline{\text { Programa para Maiores }}$ & $\begin{array}{c}50 \text { o } 55 \text { años dependiendo } \\
\text { del programa al que se } \\
\text { acojan }\end{array}$ \\
\hline
\end{tabular}

Tabla núm. 2 (Elaboración propia)

Algunas de las Universidades que han optado por mantener la enseñanza presencial en el Aula de Mayores han establecido limitaciones, con el objetivo de cumplir las restricciones sanitarias. En el caso de la Universidad de Cádiz se ha optado por mantener la docencia presencial, pero sin que se admitan alumnos de nuevo ingreso, tratando así de hacer frente a las limitaciones de aforo. Las razones que han llevado a los responsables de estos programas a mantener la enseñanza presencial han sido diversas, pero el intento de evitar la brecha digital (que en los colectivos de edad es mayor) ha jugado un papel relevante, sin duda.

c) Un tercer bloque lo constituyen las Universidades que han optado por un modelo mixto y/o híbrido (de enseñanza presencial y/o virtual, de conformidad con la evolución de la situación sanitaria):

\begin{tabular}{|c|c|c|c|}
\hline $\begin{array}{c}\text { Nombre } \\
\text { Universidad }\end{array}$ & $\begin{array}{l}\text { Nombre del } \\
\text { Programa }\end{array}$ & $\begin{array}{l}\text { Edad mínima } \\
\text { matriculados }\end{array}$ & $\begin{array}{l}\text { Solución } \\
\text { pandémica }\end{array}$ \\
\hline A Coruña & $\frac{\frac{\text { Universidade Sénior }}{\text { da Universidade da }}}{\text { Coruña (UDC) }}$ & 50 años & $\begin{array}{l}\text { Programa bimodal } \\
\text { (teleformación } \\
\text { presencial) }\end{array}$ \\
\hline Alicante & $\frac{\text { Universidad }}{\underline{\text { Permanente }}}$ & 50 años & $\begin{array}{l}\text { Alguna actividad } \\
\text { presencial, anulación } \\
\text { de numerosos cursos } \\
\text { y bimodal }\end{array}$ \\
\hline Barcelona & $\frac{\text { Universidad de la }}{\underline{\text { Experiencia }}}$ & 55 años & $\begin{array}{l}\text { Virtual el primer año } \\
\text { (mixta en los } \\
\text { siguientes) }\end{array}$ \\
\hline Lleida & Programa Sènior & 50 años & Presencial y virtual \\
\hline Salamanca & $\frac{\text { Interuniversitario de }}{\underline{\text { la Experiencia }}}$ & $\begin{array}{l}\text { Jubilados, } 55 \text { años o } \\
\text { a partir de } 50 \text { si no se } \\
\text { cubren las plazas }\end{array}$ & $\begin{array}{l}\text { Se inició presencial y } \\
\text { se pasó a virtual ante } \\
\text { la situación sanitaria }\end{array}$ \\
\hline
\end{tabular}

Tabla núm. 3 (Elaboración propia) 
Como puede verse, el número de Universidades que se han decantado por un modelo mixto, donde se combinó la enseñanza presencial y virtual, o bien se pasó de una a otra a consecuencia del empeoramiento de la situación sanitaria, es bastante más limitado que el uso exclusivo de la virtualidad o la presencialidad, tal y como se ha visto en los apartados a) y b) anteriores. De lo que no cabe duda es que la COVID-19 ha producido distorsiones importantes en la forma de llevar a cabo esta labor de la Universidad, llevando incluso a suspensiones, aplazamientos y/o cancelaciones de la misma, como se muestra en el apartado siguiente.

d) Universidades que han optado por el aplazamiento, suspensión temporal y/o cancelación del Aula de Mayores durante el curso 2020/21:

\begin{tabular}{|c|c|c|c|}
\hline $\begin{array}{c}\text { Nombre } \\
\text { Universidad }\end{array}$ & $\begin{array}{l}\text { Nombre del } \\
\text { Programa }\end{array}$ & $\begin{array}{l}\text { Edad mínima } \\
\text { matriculados }\end{array}$ & $\begin{array}{l}\text { Solución } \\
\text { pandémica }\end{array}$ \\
\hline Burgos & $\frac{\text { Interuniversitario de }}{\underline{\text { la Experiencia }}}$ & 50 años & $\begin{array}{l}\text { Se aplaza a enero de } \\
2021 ; \text { dependiendo } \\
\text { de las sedes } \\
\text { presencial o virtual }\end{array}$ \\
\hline $\begin{array}{l}\text { Católica Sta. Teresa } \\
\text { de Jesús de Ávila }\end{array}$ & $\frac{\underline{\text { Programa }}}{\frac{\text { Interuniversitario de }}{\text { la Experiencia }}}$ & 55 años & $\begin{array}{l}\text { Aplazado su } \\
\text { comienzo hasta enero } \\
\text { de } 2021\end{array}$ \\
\hline Jaume I & $\frac{\text { Universitat per a }}{\underline{\text { Majors }}}$ & 55 años & $\begin{array}{lc}\text { Primer } & \text { curso } \\
\text { aplazado; } & \text { semi- } \\
\text { presencial y virtual }\end{array}$ \\
\hline León & $\frac{\text { Interuniversitario de }}{\underline{\text { la Experiencia }}}$ & 55 años & $\begin{array}{ll}\begin{array}{l}\text { Pospuesto hasta } \\
\text { enero de } \\
\text { (presencial) }\end{array} & \end{array}$ \\
\hline $\begin{array}{l}\text { Pontificia de } \\
\text { Salamanca }\end{array}$ & $\frac{\text { Interuniversitario de }}{\underline{\text { la Experiencia }}}$ & 50 años & $\begin{array}{l}\text { Aplazada a enero, } \\
\text { cursos presenciales o } \\
\text { virtuales } \\
\text { dependiendo de la } \\
\text { sede }\end{array}$ \\
\hline Rovira i Virgili & $\frac{\frac{\text { Aules d'Extensió }}{\text { Universitària de la }}}{\text { Gent Gran }}$ & Tercera edad & $\begin{array}{ll}\text { Suspendida } & \text { la } \\
\text { docencia } & \end{array}$ \\
\hline Valencia & $\underline{\text { La Nau Gran }}$ & 55 años & $\begin{array}{l}\text { Suspendida } \\
\text { temporalmente a } \\
\text { consecuencia de la } \\
\text { situación sanitaria }\end{array}$ \\
\hline Valladolid & 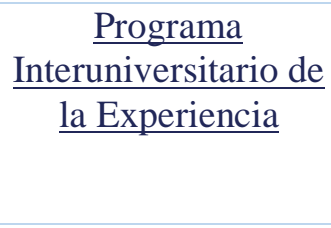 & 50 años & $\begin{array}{l}\text { Aplazada a enero, } \\
\text { cursos presenciales o } \\
\text { virtuales } \\
\text { dependiendo de la } \\
\text { sede }\end{array}$ \\
\hline Valladolid & $\frac{\begin{array}{c}\text { Universidad } \\
\text { Permanente "Millán }\end{array}}{\underline{\text { Santos" }}}$ & 40 años & $\begin{array}{l}\text { Se suspende tanto la } \\
\text { modalidad abierta } \\
\text { como el bloque de } \\
\text { especialización }\end{array}$ \\
\hline
\end{tabular}

Tabla núm. 4 (Elaboración propia)

Un total de nueve programas universitarios, más algún otro que ha optado por fórmulas mixtas y se ha citado con anterioridad, conforman este bloque, donde la 
evolución de la situación sanitaria ha provocado la necesidad de aplazar, suspender o cancelar como fórmula más drástica, los programas de formación en el Aula de Mayores. El número es significativo, pero, comparativamente hablando, supone un cambio respecto a lo acontecido en el curso académico anterior (2019/20) donde la COVID-19 impidió proseguir las actividades previamente programadas en la mayoría de las Universidades.

e) Otras situaciones diversas:

\begin{tabular}{|c|c|c|c|}
\hline $\begin{array}{c}\text { Nombre } \\
\text { Universidad }\end{array}$ & $\begin{array}{c}\text { Nombre del } \\
\text { Programa }\end{array}$ & $\begin{array}{l}\text { Edad mínima } \\
\text { matriculados }\end{array}$ & $\begin{array}{l}\text { Solución } \\
\text { pandémica }\end{array}$ \\
\hline Huelva & $\frac{\text { Aula de la }}{\text { Experiencia }}$ & 55 años & $\begin{array}{l}\text { Emite programas por } \\
\text { TV (Huelva TV) y } \\
\text { Radio (Uniradio) }\end{array}$ \\
\hline La Rioja & $\frac{\text { Universidad de la }}{\text { Experiencia }}$ & Personas adultas & $\begin{array}{l}\text { Información } \\
\text { disponible }\end{array}$ \\
\hline
\end{tabular}

Tabla 5 (Elaboración propia)

En este último caso cabe destacar la fórmula puesta en práctica por la Universidad de Huelva, emitiendo programas en la televisión (Huelva TV) y también en la radio (a través de Uniradio). Los podcasts y vídeos respectivos (o al menos un número significativo de ellos, concretamente los más recientes) están accesibles en la web del Aula de la Experiencia de dicha Universidad, lo que facilita el acceso a los mismos, si no se han podido seguir en el momento en que se emitieron. Es sin duda una iniciativa con la que se ha pretendido superar la brecha digital.

La información que ha permitido elaborar las tablas se ha obtenido acudiendo de forma individualizada y consultando la información concreta del curso 2020/21 del conjunto de Universidades socias de la Asociación Estatal de Programas Universitarios para Mayores (AEPUM), lo que ha facilitado poder investigar el programa universitario destinado a los mayores en cada una de ellas. En ocasiones esta investigación se ha tenido que desarrollar de manera más o menos intuitiva, pues el hecho de que la pandemia y su evolución sea el factor condicionante, hace que los datos proporcionados por la web de las respectivas universidades no siempre estén actualizados.

La denominación de los programas destaca por su enorme variedad (inclusive siendo algunos interuniversitarios y realizados en varios campus y/o ciudades diferentes, dependiendo de la Universidad concreta). Si bien bajo la denominación de Aula de Mayores (presente en 15 de las Universidades del listado) se tiene una idea general de los estudios a los que estamos haciendo referencia, cada Universidad les ha otorgado un nombre, que en algunos casos constituye incluso una seña de identidad. Desde Universidad Senior (denominación utilizada en 6 de ellas), Universidad de la Experiencia (en 11 de los casos citados), a algunas otras denominaciones como Peritia et Doctrina (en las Palmas de Gran Canaria), IV Ciclo (en la Universidad de Santiago), la Nau Gran (Universidad de Valencia) u otras, por citar algunos de los nombres con que se denomina esta realidad.

Respecto a los destinatarios de estos estudios cabe también hacer alusión a la diversidad reinante: así, en algunas Universidades no se determina una edad mínima como punto de partida para matricularse en estos cursos, sino que se habla de personas adultas o personas mayores; en otras se fijan los 40, 45, 50 o 55 años como edad mínima para cursar estas enseñanzas, dependiendo de cada Universidad, como puede observarse en las tablas. Otro requisito que a veces se exige es el hecho de ser residente en la 
Comunidad Autónoma de que se trate. En otras Universidades se hace mención además a las personas jubiladas, sin que sea requisito adicional tener una determinada edad si se reúne esta condición previa. En suma, dependiendo de las Universidades los criterios pueden variar, si bien la mayoría de ellas destinan estos programas, a los mayores de 5055 años. En el caso de la UMA, además, si bien originalmente el Aula de Mayores tenía simplemente esta denominación, desde hace ya algunos años se le ha añadido +55 a su nombre, como indicativo de que la misma va dirigida con carácter prioritario a este colectivo (personas de 55 años en adelante).

En relación con la adaptación de estos programas de mayores a la pandemia de la COVID-19, si bien algunas Universidades -las menos- han seguido optando por el sistema presencial, mediante el cual se impartían tradicionalmente estas enseñanzas, un número muy elevado de ellas han planteado las clases on-line (síncronas) como alternativa, teniendo presente la evolución de la situación sanitaria durante este curso académico 2020/21. Incluso, ha habido alguna Universidad, como la de Huelva, que ha optado por soluciones imaginativas como la retransmisión por televisión y por la radio de los cursos, ofreciendo un entorno accesible para todo aquel que estuviera interesado. También ha habido Universidades que han preferido posponer el comienzo de dichos cursos hasta que la situación sanitaria mejorase, suspenderlos durante este curso, suspender parcialmente alguna parte del programa, eliminar algunos cursos (usualmente los menos demandados) o incluso ofrecer programas híbridos (donde algunos cursos son presenciales y otros virtuales).

Como puede verse, cada Universidad ha adoptado las medidas más acordes a las circunstancias, tanto sanitarias como al propio desarrollo habitual de los cursos y el público al que van dirigidos. La casuística es enormemente variada y sobre todo se ha fundamentado en el realismo, la efectividad y la seguridad de un colectivo que se ha mostrado especialmente vulnerable frente a la pandemia, como es el que de manera generalizada participa en el Aula de Mayores. La idea esencial, se fundamenta en el aprendizaje durante toda la vida, factor primordial de una enseñanza universitaria dirigida a un público muy especial, en el buen sentido de la expresión.

\section{El Aula de Mayores a la luz del ODS 4: ajustes y desajustes en tiempos de COVID-19}

\subsection{El ODS 4 confrontado con el aprendizaje durante toda la vida}

Como es sabido, el ODS 4 (Martin 2016, 231) plantea en el horizonte temporal de 2015-2030, "Garantizar una educación inclusiva, equitativa y de calidad y promover oportunidades de aprendizaje durante toda la vida para todos" ${ }^{11}$. Señalamos en cursiva esa parte de la frase, que pone de relieve una de las premisas fundamentales sobre las que se asienta el Aula de Mayores: el longlife learning. Esta idea cobra aún más relevancia, si cabe, en lo que se refiere a las personas mayores (entendiéndose por tales, como hemos señalado, las personas jubiladas, mayores de 40-55 años, en ese rango que cada Universidad ha determinado respecto al público al que pretende dirigir estas enseñanzas).

Si bien existen diferentes hitos para la consecución de este Objetivo de Desarrollo Sostenible, al menos dos de ellos se encuadran plenamente entre los retos a los que la educación de adultos ha de referirse, en este contexto universitario que tratamos de analizar. Serían los siguientes:

11 Véase https://es.unesco.org/gem-report/node/1346. 
“4.3 Para 2030, asegurar el acceso en condiciones de igualdad para todos los hombres y las mujeres a una formación técnica, profesional y superior de calidad, incluida la enseñanza universitaria.

4.5 Para 2030, eliminar las disparidades de género en la educación y garantizar el acceso en condiciones de igualdad de las personas vulnerables, incluidas las personas con discapacidad, los pueblos indígenas y los niños en situaciones de vulnerabilidad, a todos los niveles de la enseñanza y la formación profesional" $" 12$.

En relación con la puesta en práctica de este ODS, la Declaración de Incheon, aprobada en el contexto del Foro Mundial sobre la Educación 2015 que tuvo lugar en esta ciudad de la República de Corea, auspiciado por la UNESCO, el UNICEF, el Banco Mundial, el Fondo de Población de Naciones Unidas (UNFPA), el PNUD, ONU-Mujeres y el ACNUR, constituye un hito destacable ${ }^{13}$. La idea que se persigue se desprende de su mismo título "Educación 2030. Hacia una educación inclusiva y equitativa de calidad y un aprendizaje a lo largo de la vida para todos", que pone el foco en esa noción inclusiva, continua a lo largo de la vida (en todos los niveles educativos) y donde las TIC juegan un papel fundamental, conforme a lo establecido en el apartado 10 de dicha Declaración, que se manifiesta en estos términos:

"Nos comprometemos a promover oportunidades de aprendizaje de calidad a lo largo de la vida para todos, en todos los contextos y en todos los niveles educativos. (...) Es preciso aprovechar las tecnologías de la información y la comunicación (TIC) para reforzar los sistemas educativos, la difusión de conocimientos, el acceso a la información, el aprendizaje efectivo y de calidad, y una prestación más eficaz de servicios".

En esta misma línea se pronuncia el Marco de Acción de Educación 2030, auspiciado en noviembre de 2015 por la UNESCO y sus Estados miembros, que trata de establecer estrategias concretas para hacer realidad los postulados de la Declaración ${ }^{14}$. Las propuestas están claras, pero cabe preguntarse si realmente la realidad que nos ha traído la pandemia, permite acoger con cierto grado de optimismo las mismas o habrán de posponerse, a la espera de tiempos mejores. En el apartado siguiente abordaremos precisamente estas ideas, siendo realistas, y teniendo presente la experiencia acumulada durante el año en curso, a la que nos hemos referido, en el Aula de Mayores+55 de la Universidad de Málaga.

\subsection{Los efectos colaterales de la pandemia para el colectivo de mayores $\mathbf{y}$ su formación}

La COVID-19 ha impactado en nuestras vidas y también lo ha hecho en el colectivo de los mayores, quizá aún con mayor crudeza, desde un punto de vista sanitario (el drama vivido en las residencias en nuestro país en la primera oleada de la pandemia es un ejemplo, entre otros muchos, de ello). Pero su incidencia ha sido radical en otros ámbitos, como el de la interacción social y el educativo, que es el sector concreto al que estamos dedicando atención. En numerosos lugares, las actividades que venían realizando los mayores a través de asociaciones, ayuntamientos, ONG, etc., simplemente han tenido que ser suspendidas y/o

\footnotetext{
12 Véase https://es.unesco.org/gem-report/node/1346. La cursiva es nuestra, poniendo énfasis en el carácter vulnerable de este colectivo, al menos a priori.

${ }^{13}$ Véase el contenido de la misma en https://unesdoc.unesco.org/ark:/48223/pf0000233813.

${ }^{14}$ Véase https://es.unesco.org/news/adopcion-del-marco-accion-educacion-2030.
} 
aplazadas, a la espera de que la situación sanitaria mejorase, teniendo presente el carácter especialmente vulnerable de este colectivo frente a la pandemia.

En este sentido, la educación para toda la vida -y concretamente la posibilidad de formarse y participar en el Aula de Mayores - ha de tener presente, como sucede en el marco educativo de manera general, el enfoque de derechos (Quintana Avello 2020, 5). De ello se derivan una serie de características, enfatizadas por UNICEF y la UNESCO (disponibilidad, accesibilidad -que incluye la no discriminación, la accesibilidad material y la accesibilidad económica-, aceptabilidad y adaptabilidad), que han de estar presentes siempre y, a nuestro entender, aún más en este contexto que hemos vivido recientemente.

Sin duda, la llegada de la pandemia y el confinamiento en España provocó la cancelación de las actividades previstas en el Aula de Mayores de la mayoría de las Universidades, al menos durante lo que restaba del curso académico. Ni el sistema de impartición de la docencia, ni los docentes, ni el alumnado estábamos preparados para ese cambio inesperado que se produjo ante la crisis sanitaria. El cierre de las Universidades fue una consecuencia directa de ello, pues la presencialidad y la socialización han sido dos de las ideas eje conformadoras de estas experiencias.

Y como correlato lógico, al transcurrir el tiempo y dada la imposibilidad de comenzar el curso de manera habitual en el Aula de Mayores, era prácticamente ineludible dar el salto a la virtualidad en muchas de las Universidades españolas. En otras se suprimieron o aplazaron las actividades habituales, se compaginó lo presencial y lo virtual, o incluso se buscaron mecanismos alternativos para superar la brecha digital de este colectivo. Si esta brecha ya existía (Martínez Heredia y Rodríguez García 2018, 37), la pandemia no ha hecho más que agudizarla de manera general, y más aún en este grupo de personas de edad, no tan familiarizado como los jóvenes con las tecnologías (Rodicio-García et al.2020, 107).

El disfrute de todos los derechos humanos por las personas de edad es un reto de nuestras sociedades, y la situación que vivimos no ha hecho más que poner el acento en la necesidad de tener presente esta realidad, al igual que la que atañe a todos los colectivos especialmente vulnerables, de los que las personas con diversidad funcional conforman un amplio sector necesitado de atención. Y en múltiples ocasiones coinciden ambas situaciones (edad y diversidad funcional). La toma de conciencia a nivel global de estas realidades está comenzando, y valga como muestra la opinión vertida por Michelle Bachelet, Alta Comisionada de Naciones Unidas para los Derechos Humanos, así como del propio Secretario General de la ONU, Antonio Guterres, a este respecto ${ }^{15}$.

Sin duda alguna, la COVID-19 ha provocado en materia educativa una crisis ${ }^{16}$, perturbación ${ }^{17}$, aunque también se puede definir como una oportunidad (Burgos et al. 2020, 7-9; Cotino Hueso, 2020, 18; Marco, 2020, 1). Las nociones de resiliencia, prevención y gestión de crisis (Aberle y Hoekstra, 2020; Bautista Hernáez, 2021) han salido también a relucir en el contexto actual, donde la educación, al igual que todas las facetas de la vida, se han visto afectadas de un modo u otro. La Universidad, de manera general y la Universidad para Mayores, de forma específica, no podían permanecer ajenas a esta realidad. Ese paso a lo

\footnotetext{
${ }^{15}$ Véase https://www.ohchr.org/SP/NewsEvents/Pages/DisplayNews.aspx?NewsID=25879\&LangID=S.

${ }^{16}$ Véase https://www.ohchr.org/EN/NewsEvents/Pages/COVID-19-and-education.aspx.

${ }^{17}$ Véase https://es.unesco.org/news/ano-educacion-perturbada-covid-19-como-esta-situacion.
} 
virtual ha llevado a algunos autores a repensar este contexto y su vuelta a la "normalidad", utilizando la expresión "universidad de baja densidad" (Maloney y Kim, 2020). Pros y contras (Bonilla Guachamín, 2020) se unen en este escenario educativo, marcado por la evolución de la pandemia y sus consecuencias sanitarias, cuyo desenlace y consecuencias definitivas aún están por escribir.

\section{A modo de colofón, ante una realidad en transición.}

Aún es muy pronto para evaluar las consecuencias que la COVID-19 ha tenido para el derecho a la educación (Ruiz, 2020), de manera general y para la educación a lo largo de toda la vida, como premisa básica y fundamental sobre la que se asienta el Aula de Mayores en las instituciones universitarias. Pero, qué duda cabe que ha afectado de lleno a la forma en que se imparten dichas enseñanzas e inclusive, a los eventuales participantes en las mismas, especialmente cuando tienen lugar en un contexto necesariamente virtual. Vivimos tiempos caracterizados por la provisionalidad (Murillo y Duk 2020), con lo cual debemos necesariamente volver a reflexionar sobre las lecciones que nos ha dejado la pandemia, cuando esta situación pase, aunque seguramente sus efectos (positivos y negativos) perdurarán en el tiempo.

En general, puede afirmarse en estos tiempos de provisionalidad, que la mayor parte de las Universidades españolas (tanto públicas como privadas) que venían impartiendo, como parte de su labor social, formación a las personas mayores, han continuado su labor, pese a todo. Por supuesto, la adaptación ha sido imprescindible, y respecto a ello las opciones también han sido variadas, si bien en un porcentaje muy elevado de casos se ha acudido a la fórmula virtual (con clases sincrónicas). Ha sido una apuesta, sin duda arriesgada, puesto que no debemos olvidar que la brecha digital es una realidad. Sin duda, esta no es más que una valoración preliminar, inmersos como estamos aún en esta situación alarmante que llevamos viviendo desde el 2020.

Por supuesto, la Universidad para Mayores debe seguir siendo esa universidad equitativa e inclusiva que permita que todos accedan a ella, pero casi con total seguridad la vuelta a las aulas ya no volverá a ser como antes. Realizar vaticinios en estos tiempos de mudanza es arriesgado, pero quizá no sea una osadía pensar que también la virtualidad pasará a ser un escenario más -complementario, sin lugar a dudas- en la educación a lo largo de toda la vida, y en especial del colectivo de las personas mayores, que han dado ejemplo de resiliencia como los que más. A ellos, a todas esas personas de edad que, pese a las difíciles circunstancias, no han dudado en seguir con sus propósitos de formarse y continuar en ese camino, van dedicadas estas reflexiones. 


\section{Referencias bibliográficas y otros documentos de consulta}

a) Monografías y artículos:

- $\quad$ ABERLE, N. y HOEKSTRA, M.M. (2020), Resilience Based Crisis Management in Public Educational Institutions at the Time of Global Pandemic of COVID-19: The Implications for Ensuring SDG4, en http://mau.divaportal.org/smash/record.jsf?pid=diva2\%3A1472829\&dswid=-5220.

- BAUTISTA-HERNÁEZ, A. (2021), Derecho Internacional y Gestión de Catástrofes: Grandes Avances y Retos Pendientes, Tirant lo Blanch, Valencia.

- BONILLA GUACHAMÍN, J.A. (2020), "Las dos caras de la educación en el COVID-19”, $\quad$ CienciAmérica, vol. 9 en http://201.159.222.118/openjournal/index.php/uti/article/view/294/461, 10 pp.

- BURGOS, D., TLILI, A. y TABACCO, A. (eds.) (2020), Radical Solutions for Education in a Crisis Context. COVID-19 as an Opportunity for Global Learning, "Education in a Crisis Context: Summary, Insights and Future", capítulo de estos mismos autores, Springer, Singapur, Berlín, pp. 3-10.

- COTINO HUESO, L. (2020), "La enseñanza digital en serio y el derecho a la educación en tiempos del coronavirus", Revista de Educación y Derecho, n. 21, 29 pp.

- GAIRÍN, J., RODRIGUEZ-GÓMEZ, D., ARMENGOL. C. y DEL ARCO, I. (2013), "El acceso a la Universidad para personas mayores en España. Los programas universitarios para personas mayores: revisando la realidad", Revista de Orientación Educacional, v. 27, núm. 51, pp. 45-65.

- MALONEY, E.J. y KIM, J. (2020), The Low-Density University: 15 Scenarios for Higher Education, Johns Hopkins University Press, Baltimore.

- MARTIN, J.P. (2016), "SDG 4: Lifelong, Inclusive and Equitable Education: Goal 4", en International Society and Sustainable Development Goals, DURÁN y LALAGUNA, P., DÍAZ BARRADO, C.M. y FERNÁNDEZ LIESA, C. (eds.), Aranzadi Thomson Reuters, Madrid, pp. 231-247.

- MARCO, B. (2020), "Coronavirus, COVID-19 and the Right to Education. Lessons to be Learned (A Contribution to Good Practices)", en https://www.ohchr.org/_layouts/15/WopiFrame.aspx? sourcedoc=/Documents/Issue s/Education/COVID19/BertaMarco.docx\&action=default\&DefaultItemOpen=1， 5 pp.

- MARTÍNEZ HEREDIA, N. y RORÍGUEZ- GARCIA, A.-M. (2018), "Alfabetización y competencia digital en personas mayores: el caso del aula permanente de formación abierta de la Universidad de Granada (España)", Revista Espacios, vol. 39, núm. 10, p. 37, accesible en http://revistaespacios.com/a18v39n10/a18v39n10p37.pdf.

- $\quad$-MORENO CRESPO, P.A. y CRUZ DÍAZ, M.R. (2012), "Promoción de la calidad de vida a través de los programas socioeducativos para mayores, Universidad y Mayores", I Congreso Virtual Internacional sobre Innovación Pedagógica y Praxis Educativa, Innovagogia 2012, Libro de Actas, 21, 22 y 23 de noviembre de 2012, pp. 1522-1533, accesible en https://www.upo.es/cms1/export/sites/upo/aulamayores/cuadernos/documentos/promocion_calidad_vida.pdf.

- $\quad$-MURILLO, F.J. y DUK, C. (2020), "El Covid-19 y las brechas educativas", Revista Latinoamericana de Educación Inclusiva, vol. 14, núm. 1, accesible en https://scielo.conicyt.cl/scielo.php?pid=S0718-

$73782020000100011 \&$ script=sci_arttext. 
- QUINTANA AVELlO, I. (2020), "Covid-19 y Cierre de Universidades. ¿Preparados para una educación a distancia de calidad?”, Revista Internacional de Educación para la Justicia Social, vol. 9, núm. 3 extraordinario, 11 pp., accesible en https://revistas.uam.es/riejs/article/view/12232/12094.

- RODICIO-GARCÍA, M.L., RIOS-DE-DEUS, M.P., MOSQUERA-GONZÁLEZ, M.J. y PENADO ABILLEIRA, M., "La Brecha Digital en Estudiantes Españoles ante la Crisis de la Covid-19", Revista Internacional de Educación para la Justicia Social, vol. 9, núm. 3 extraordinario, pp. 103-125, en https://revistas.uam.es/riejs/article/view/riejs2020_9_3_006/12444.

- RUIZ. G.R. (2020), "Marcas de la pandemia: el derecho a la educación afectado", Revista Internacional de Educación para la Justicia Social, vol. 9, núm. 3 extraordinario, pp. 45-59, en https://ri.conicet.gov.ar/bitstream/handle/11336/108882/CONICET_Digital_Nro.fa d2c5dc-48ef-40e2-b804-c53a7536fc86_A.pdf?sequence=2\&isAllowed=y.

- TORRES CAZORLA, M.I. (2001), "La sociedad internacional contemporánea: problemas recientes. Una aproximación a la crisis de las Naciones Unidas en los albores del siglo XXI", Aula de Formación Abierta 2000, Universidad para mayores, pp. 181-188; (2002) "Las repercusiones de los acontecimientos del 11 de septiembre de 2001 en la sociedad internacional contemporánea", Aula de Formación Abierta 2002, Universidad para mayores, pp. 293-299; (2003) "La extradición en Derecho Internacional", Aula de Formación Abierta 2002/2003, Universidad para mayores, pp. 403-408.

- VALLE APARICIO, J.E. (2014), "Educación permanente: los programas universitarios para mayores en España como respuesta a una nueva realidad social", Revista de la Educación Superior, vol. XLIII (3), n. 171, julio-septiembre 2014, pp. 117-138.

b) Otros documentos de consulta:

- $\quad$ BOE (Sección dedicada a la COVID-19): https://www.boe.es/biblioteca_juridica/index.php?tipo=C.

- UNESCO (Organización de Naciones Unidas para la Educación, la Ciencia y la Cultura):

- Información sobre el ODS 4: https://es.unesco.org/gem-report/node/1346

- Declaración de Incheon: http://www.unesco.org/new/fileadmin/MULTIMEDIA/HQ/ED/ED_new/pdf/FF A-ENG-27Oct15.pdf.

- Marco de Acción Educativa 2030: https://es.unesco.org/news/adopcion-delmarco-accion-educacion-2030. 